\title{
Genomic signatures of different adaptations to environmental stimuli between wild and cultivated Vitis vinifera L
}

\author{
Annarita Marrano (1)', Diego Micheletti ${ }^{2}$, Silvia Lorenzi ${ }^{1}$, David Neale ${ }^{3}$ and M. Stella Grando ${ }^{1,4}$
}

\begin{abstract}
The application of population genetic methods in combination with gene mapping strategies can help to identify genes and mutations selected during the evolution from wild plants to crops and to explore the considerable genetic variation still maintained in natural populations. We genotyped a grapevine germplasm collection of 44 wild (Vitis vinifera subsp. sylvestris) and 48 cultivated ( $V$. vinifera subsp. sativa) accessions at $54 \mathrm{~K}$ single-nucleotide polymorphisms (SNPs) to perform a whole-genome comparison of the main population genetic statistics. The analysis of Wright Fixation Index $\left(F_{S T}\right)$ along the whole genome allowed us to identify several putative "signatures of selection" spanning over two thousand SNPs significantly differentiated between sativa and sy/vestris. Many of these genomic regions included genes involved in the adaptation to environmental changes. An overall reduction of nucleotide diversity was observed across the whole genome within sylvestris, supporting a small effective population size of the wild grapevine. Tajima's D resulted positive in both wild and cultivated subgroups, which may indicate an ongoing balancing selection. Association mapping for six domestication-related traits was performed in combination with population genetics, providing further evidence of different perception and response to environmental stresses between sativa and sylvestris.
\end{abstract}

\section{Introduction}

The Eurasian grape (Vitis vinifera L.) is one of the most important crops worldwide for its global distribution and economic value ${ }^{1}$. $V$. vinifera $L$. exists as the cultivated form $V$. vinifera subsp. sativa (or vinifera; hereafter called sativa) and the wild-form $V$. vinifera subsp sylvestris (hereafter called sylvestris), which is assumed to be the ancestor of modern cultivars. The two subspecies exhibit several phenotypical differences, notably in flower sex, seed shape, bunch and berry size, and leaf morphology ${ }^{2}$. In particular, sylvestris is dioecious with separate male and female individuals, and in general produces few bunches with small, black and juiceless berries. On the contrary,

Correspondence: Annarita Marrano (marrano.annarita@gmail.com)

'Department of Genomics and Biology of Fruit Crops, Research and Innovation Centre, Fondazione Edmund Mach, San Michele all 'Adige (TN), Italy

${ }^{2}$ Computational Biology Unit, Research and Innovation Centre, Fondazione Edmund Mach, San Michele all 'Adige (TN), Italy

Full list of author information is available at the end of the article sativa presents hermaphroditic flowers and enormous phenotypic variability regarding number, size, taste, and colour of the fruit. Previous surveys in grapevine collections have also outlined weak but clear genetic differentiation among cultivated and ex situ wild accessions ${ }^{3-5}$. The cultivated grapevine has broad genetic variation, which is likely the result of sexual reproduction, vegetative propagation, and somatic mutations occurring during the long history of grapevine cultivation ${ }^{1}$. In contrast, sylvestris is less diverse than the domesticated form ${ }^{6}$. The present distribution of the wild vinifera is fragmented in relict populations with very few individuals. The decline of sylvestris has drastically increased over the last two centuries because of the introduction of pests and diseases (phylloxera, downy and powdery mildew) from North America, and the anthropic impact on wild grapevine habitats $^{7}$. Also, gene flow between cultivated and wild grapevines might lead to domestic introgression and genetic loss in the small relict populations of sylvestris ${ }^{8}$.

\section{(c) The Author(s) 2018}

(c) (i) Open Access This article is licensed under a Creative Commons Attribution 4.0 International License, which permits use, sharing, adaptation, distribution and reproduction cc) in any medium or format, as long as you give appropriate credit to the original author(s) and the source, provide a link to the Creative Commons license, and indicate if changes were made. The images or other third party material in this article are included in the article's Creative Commons license, unless indicated otherwise in a credit line to the material. If material is not included in the article's Creative Commons license and your intended use is not permitted by statutory regulation or exceeds the permitted use, you will need to obtain permission directly from the copyright holder. To view a copy of this license, visit http://creativecommons.org/licenses/by/4.0/. 
Recently, several efforts have been devoted to the study of biotic and abiotic stresses-response in wild $V$. vinifera, revealing accessions tolerant to salt stress ${ }^{9}$, lime-induced chlorosis $^{10}$ and downy mildew (Plasmopara viticola) $)^{11}$. These findings shift sylvestris into the center of attention as a valuable genetic resource for grapevine resilience breeding, which has been so far based on the exploitation of the innate resistance/tolerance of other Vitis species. The identification of genetic resistance in sylvestris may allow preserving the vinifera genomic background of high fruit quality in future breeding cycles. The need to investigate the genetic diversity of sylvestris becomes more significant considering the potential increased vulnerability to environmental changes and the appearance of new pests and diseases since the limited number of grape cultivars grown worldwide.

The morphological and genetic divergence between the two vinifera subspecies may be the result of different evolutionary forces acting on sativa in the vineyards and on sylvestris in floodplain forests. Whole-genome comparison of genetic diversity is a feasible strategy to discover the genes involved in this progressive and subtle differentiation between sativa and sylvestris ${ }^{12}$. Genome scanning for signatures of selection has been reported for several crops such as tomato ${ }^{13}$, maize ${ }^{14}$, rice ${ }^{15}$ and barrel medic $^{16}$. In grapevine, Myles et al. ${ }^{4}$ used $9 \mathrm{~K}$ SNPs to compare the haplotype diversity between sativa and sylvestris, identifying a $5-\mathrm{Mb}$ putative signature of selection on chromosome 17. Association mapping (AM) is an alternative to population genetics to uncover the genomic regions responsible for the phenotypic variation observed in $V$. vinifera. However, few studies of AM in fruit species have been reported so far, due to the difficulties in building up an ideal large association panel without an intricate pattern of population stratification and familial relatedness ${ }^{17}$. Chitwood et al. ${ }^{18}$ performed a genome-wide association scan (GWAS) to map the genetic basis of leaf morphology in grapevine, identifying a handful of SNPs associated with just four of the 13 phenotyped traits. This GWA study underlined the limited power of AM in grapevine, which can mainly be attributed to its rapid linkage disequilibrium (LD) decay ${ }^{19}$. More recently, Migicovsky et al. ${ }^{20}$ combined GWAS with selective sweep mapping to deal with the fast LD decay and increase the power of detecting loci targeted during the domestication and breeding of wine and table grapes.

In the present research, we assess the distribution and magnitude of the genomic differences between two populations of wild and domesticated grapevine. The study is organized into the following two main milestones, (i) the development of high-density SNP-based genotyping for a germplasm collection, including many authentic wild $V$. vinifera; (ii) the whole-genome scan for signatures of selection by using a combination of population genetics methods. Our results provide evidence of genetic differentiation between sativa and sylvestris individuals at genomic regions mainly involved in response to environmental stimuli. These findings draw attention to wild grapevines as a valuable source of resilience factors, whose re-discovery might be fundamental for sustainable agriculture in the future.

\section{Materials and methods \\ SNP genotyping of a grapevine germplasm population}

A germplasm collection of 48 cultivated (Vitis vinifera spp. sativa) and 44 wild female (Vitis vinifera spp. sylvestris) grapevines (Supplementary Table S1) was sorted at the FEM grape repository (ITA362) as described by Marrano et al. $^{6}$. All samples were grafted on the rootstock Kober $5 \mathrm{BB}$, and uniformly pruned and trained according to the Guyot system. DNA extraction was performed from young leaf tissue of one field grown plant per accession using the DNeasy 96 plant mini kit (QIAGEN, Germany). Both the Synergy HT Multi-Mode Microplate Reader (BioTek) and the NanoDrop 8000 UV-Vis Spectrophotometers (Thermo Scientific) were used to inspect DNA concentration and purity. DNA samples were adjusted to a minimum concentration of $100 \mathrm{ng} / \mu \mathrm{L}$ in $10 \mu \mathrm{L}$ aliquots. The commercial GrapeReseq $20 \mathrm{~K}$ SNPs array (http://urgi.versailles.inra.fr/ Species/Vitis/GrapeReSeq_Illumina_20K) ${ }^{21}$ was used to genotype the whole population with the Infinium technology according to the Illumina protocol (Illumina, Inc., San Diego, CA, USA). The genomic DNA of the Pinot Noir cultivar was used as a control. SNPs genotypes were scored using the Genotyping Module v1.9 of the Illumina GenomeStudio Data Analysis software. SNPs with a Call Freq score 0 and a GenTrain $<0.6$ were filtered out. Markers with a Cluster Sep score $<0.4$ were visually inspected for accuracy of the SNP calling. SNPs with R mean score $>0.3$ and with clusters not overlapped were retained. The obtained high-quality SNPs were merged in a unique panel with $37 \mathrm{~K}$ SNPs from a RAD-seq data set previously generated $^{6}$. For the SNPs shared between the two experiments, only the SNP profiles from the $20 \mathrm{~K}$ Illumina array were retained. Samples and SNP loci with a call rate $<0.8$ were filtered out. Genotype imputation was performed to fill in missing data using LinkImpute v1.1.1 software, which is based on a $k$-nearest neighbour genotype imputation method (LD-kNNi) designed to work with unordered markers $^{22}$. Finally, SNPs with a minor allele frequency $(\mathrm{MAF})<0.05$ were removed using Plink v1.9 software $^{23}$.

\section{Analysis of population structure}

The genetic structure of the germplasm population was analysed with fastSTRUCTURE v1.0 ${ }^{24}$. A number of ancestral genetic groups (K), ranging from 1 to 10 , was tested by ten independent iterations for each $\mathrm{K}$. The most likely $K$ value was chosen running the algorithm for 
Table 1 Summary of SNPs filtering after the population genotyping assays with the Vitis20K Illumina chip and RAD-seq approaches

\begin{tabular}{lllll}
\hline Genotyping technology & Initial No. of SNPs & $\begin{array}{l}\text { No of SNPs removed for a missing rate } \\
\text { > }\end{array}$ & $\begin{array}{l}\text { No of SNPs removed for a MAF } \\
\mathbf{0 . 0 5}\end{array}$ & Final No of SNPs \\
\hline Vitis20K & 16,563 & 338 & 3600 & 12,625 \\
RAD-seq & 37,594 & 21,920 & 1330 & 14,268 \\
Total & 54,157 & 22,258 & 4930 & 26,893 \\
\hline
\end{tabular}

multiple choices of $K$ and by plotting the marginal likelihood of the data. The software CLUMPP v1.1.2 ${ }^{25}$ was used to find optimal alignments of the independent runs and the output was used directly as input into the program for cluster visualisation DISTRUCT v1.1. ${ }^{26}$. Moreover, a Principal Component Analysis (PCA) was performed as implemented in 'adegenet ${ }^{27} \mathrm{R}$ package for the multivariate analysis of genetic markers.

\section{LD decay}

Linkage disequilibrium (LD) was estimated between all SNPs with a MAF $>5 \%$ in the whole germplasm population and within sativa and sylvestris subgroups separately by using Plink v1.9 software ${ }^{23}$. The classical $r^{2}$ estimate of the correlation between genotypes was used. LD decay was explored by plotting the median $r^{2}$ in sequential bins of $10 \mathrm{~Kb}$ against the physical position. Moreover, LD landscape of each chromosome was also inspected through heat-map visualisation with the software Haploview v4.1 $1^{28}$.

\section{Genomic differentiation between sativa and sylvestris genotypes}

Fixation index $\left(F_{\mathrm{ST}}\right)$ was measured between sativa and sylvestris accessions with VCFtools v0.1.13 ${ }^{29}$, setting a sliding window of $100 \mathrm{~kb}$ with a step size of 10 $\mathrm{kb}$. Genomic windows with the top $5 \%$ of $\mathrm{F}_{\mathrm{ST}}$ values were selected as candidate regions for further analysis. To verify the empirical cutoff with low false discovery rate, we performed whole-genome permutation tests to ascertain the thresholds for identifying genomic regions highly differentiated between the two grapevine subgroups. In more detail, all the genotypes of sativa and sylvestris were shuffled, and the $F_{\mathrm{ST}}$ analysis was performed with the same parameters 1000 times. Nucleotide diversity $(\pi)$ and Tajima's $D^{30}$ were estimated along the whole genome in 100-kb windows with a step size of $10 \mathrm{~kb}$ using VCFtools, to interpret better the results gained with the $F_{\mathrm{ST}}$ analysis and clarify how sativa and sylvestris genotypes differentiated. The grape gene annotation v2.1 hosted on http://genomes.cribi.unipd.it/grape $/{ }^{31}$ was used to investigate the putative gene functions of the genomic regions with the top $5 \%$ of $F_{\mathrm{ST}}$ values. In particular, the distribution of the identified genes into different biological processes was evaluated using the weight01 method provided by the $\mathrm{R}$ package topGO ${ }^{32}$. The Kolmogorov-Smirnov-like test was performed to assess the significance of over-representation of GO categories compared with all genes in the grapevine gene prediction. Also, differentiation in the genomic regions reported in the literature associated with flower and fruit traits was checked.

\section{Association mapping for domestication-related traits}

Genotype-phenotype associations were tested for up to six domestication-related traits (single bunch weight $(\mathrm{SBCW})$, single berry weight (SBW), yield, number of bunches per plant (NBCs), total soluble solids $\left(B\right.$ rix $\left.^{\circ}\right)$ and $\mathrm{pH}$; see Supplementary Note S1), using both genotypic best linear unbiased predictors (BLUPs) and the average performance of each sample in each year separately. Also, GWAS was run for the "Species" trait coded as a binary phenotype assigning 1 to sativa accessions and 0 to sylvestris samples. GWAS was carried out by applying three models, which account for different confounding factors to avoid spurious marker-trait associations (Supplementary Note S1). All three models are implemented in TASSEL v5.0 software ${ }^{33}$. A quantile-quantile (Q-Q) plot was used to choose the model that better fit population structure and familial relatedness in the marker-trait association (Supplementary Note S1). $P$ values adjustment for multiple testing was performed, and the Bonferronicorrected critical $p$ values and False Discovery Rate (FDR) were used to identify significant marker-trait associations. Manhattan plots were displayed accordingly using the 'qqman' v0.1.3 $\mathrm{R}$ package ${ }^{34}$. The positions of markers significantly associated with phenotypes were used to investigate the grapevine gene annotation v2.1. With regard to the extent of LD, windows of $10 \mathrm{~kb}$ upstream and downstream, the SNPs of interest were used to identify candidate genes. If the markers fell within long LD blocks, the entire genomic region located between the extreme SNPs was explored. 


\section{Results and discussion}

SNP genotyping of a grapevine germplasm population

A total of 92 wild and domesticated grapevine accessions were genotyped using the custom Illumina Infinium Vitis20K SNP array and a novel RAD-seq approach ${ }^{6}$. We merged the two SNPs matrices in a unique panel since the distribution of allele frequencies within the sativa and sylvestris subgroups showed the same trend (Supplementary Figure S1). The merged data set included totally 54,157 SNPs (Table 1). We first filtered for a missing rate $>0.2$, removing six samples and 22,258 markers (Supplementary Table S1). As shown in Table 1, most of the SNPs filtered out due to high missing rate came from RAD-seq. This result is a common issue of all methods of reduce representation sequencing, where several technical factors led all the sequenced regions not to be evenly covered in all the individuals of the population ${ }^{35}$. After imputing the remaining missing genotypes, SNPs with a minor allele frequency (MAF) $<0.05$ were removed. Most of the SNPs with a low MAF came from the Vitis20K array (Table 1), and they probably resulted from errors in the genotype calling. The final panel counted 86 samples and 26,893 SNPs (hereafter called $26 \mathrm{~K} \mathrm{SNPs)} \mathrm{with} \mathrm{an}$ average of $1.3 \mathrm{~K}$ SNPs per chromosome. In particular, the SNP density ranged from one SNP every $15 \mathrm{~kb}$ on chr8 to one SNP every $21 \mathrm{~kb}$ on chr19.

\section{Analysis of population structure}

We used the $26 \mathrm{~K}$ SNPs panel to investigate the population structure and visualise the relationships among individual accessions using two different approaches: Principal Component Analysis (PCA) and model-based clustering. Figure 1 shows the first two principal components (PCs), which accounted for the $21 \%$ of the total variance. $\mathrm{PC} 1$ differentiates sylvestris genotypes from the cultivated varieties, whereas PC2 reflects the variability among sativa accessions.

As a second approach, we used the clustering algorithm implemented in fastSTRUCTURE software (Fig. 2). The optimal number of subgroups was three, with $81 \%$ of the individuals showing a clear assignment to a cluster (membership likelihood > 0.75; Supplementary Table S2). The two principal groups included 28 sativa accessions and 36 sylvestris individuals, respectively, while Pinot Noir, Gewürtztraminer (an aromatic mutation of Traminer Rot) and Mornan Noir cultivars clustered together in a third separate group. Previous studies with microsatellite markers (SSRs) ${ }^{36}$ have already suggested the firstdegree relationship of Pinot Noir and Traminer. Moreover, Pinot Noir and Traminer have presumably ancient origins, and many modern cultivars are their first-degree relatives $^{37}$. Indeed, many of the 19 genotypes (13 sativa and six sylvestris) not assigned to a defined group by fastSTRUCTURE exhibited admixture with this small cluster (K2, Supplementary Table S2). However, the analysis of the population structure highlighted how sativa and sylvestris individuals were well distinguished as two separated groups with a low level of admixture. This result is consistent with previous reports based on SSR and SNP genetic profiles, which showed a clear distinction between wild and cultivated individuals ${ }^{5}$. However, we used sylvestris individuals previously clustered through a hierarchical STRUCTURE analysis, and sativa accessions selected from a core collection that maximises the genetic diversity present in the whole germplasm collection ${ }^{6}$. Therefore, biases in allele frequencies may have been introduced, leading to an underestimation of the real level of admixture between the two subspecies.

\section{Estimation of linkage disequilibrium}

We used the $26 \mathrm{~K} \mathrm{SNPs}$ data set to estimate and validate the level of LD along the whole genome within the investigated germplasm collection of grapevine ${ }^{6}$. The LD, as measured by the standard $\mathrm{r}^{2}$ correlation coefficient, decayed below 0.2 within $10 \mathrm{~kb}$ (Fig. 3). Such rapid LD decay is consistent with the results of Myles et al. ${ }^{4}$, which detected a low level of LD $\left(r^{2}<0.2\right)$ at short physical distances using the Vitis9K SNP array. Lijavetzky et $\mathrm{al}^{38}$. observed an even lower level of LD, which decayed within 100-200 bp in more than 200 gene sequences. On the other hand, Nicolas et al. ${ }^{17}$ found that the decay of LD down to 0.2 ranged from 9 to $458 \mathrm{~kb}$. These discrepancies may be related to the low number of genomic regions investigated in both LD surveys compared to our genomewide analysis of LD. We confirmed the evidence of a rapid LD decay in grapevine, which is in agreement with the high polymorphic rate of the grapevine genome ${ }^{39}$.

When analysed separately in the two subspecies, the decay of LD appeared slower within the sylvestris group, where $r^{2}$ reached values below 0.2 within $20 \mathrm{~kb}$. Therefore, the increase in SNPs density throughout the genome did not have any significant contribution towards shaping the LD patterns within the two grapevine subpopulations. The longer extent of LD observed in the sylvestris subgroup can be related to an elevated level of inbreeding as well as to the common Italian origin of most of our wild accessions $^{6}$. The differences in LD extent between sativa and sylvestris accessions were more evident by the comparison of the LD patterns on each chromosome. In particular, long-range LD (LRLD) between widely separated loci on the chromosome (distance $>1 \mathrm{Mb}$ ) was observed for almost all chromosomes in the sylvestris group (Supplementary Figure S3). The presence of LRLD suggested the action of some forces, such as genetic drift, hitchhiking with positive-selected mutation or structural variation in chromosomes ${ }^{40}$. Blocks of short-range LD were also observed within sativa on chromosomes 2, 6, 17 and 18 (Supplementary Figure S2). Here major QTLs 


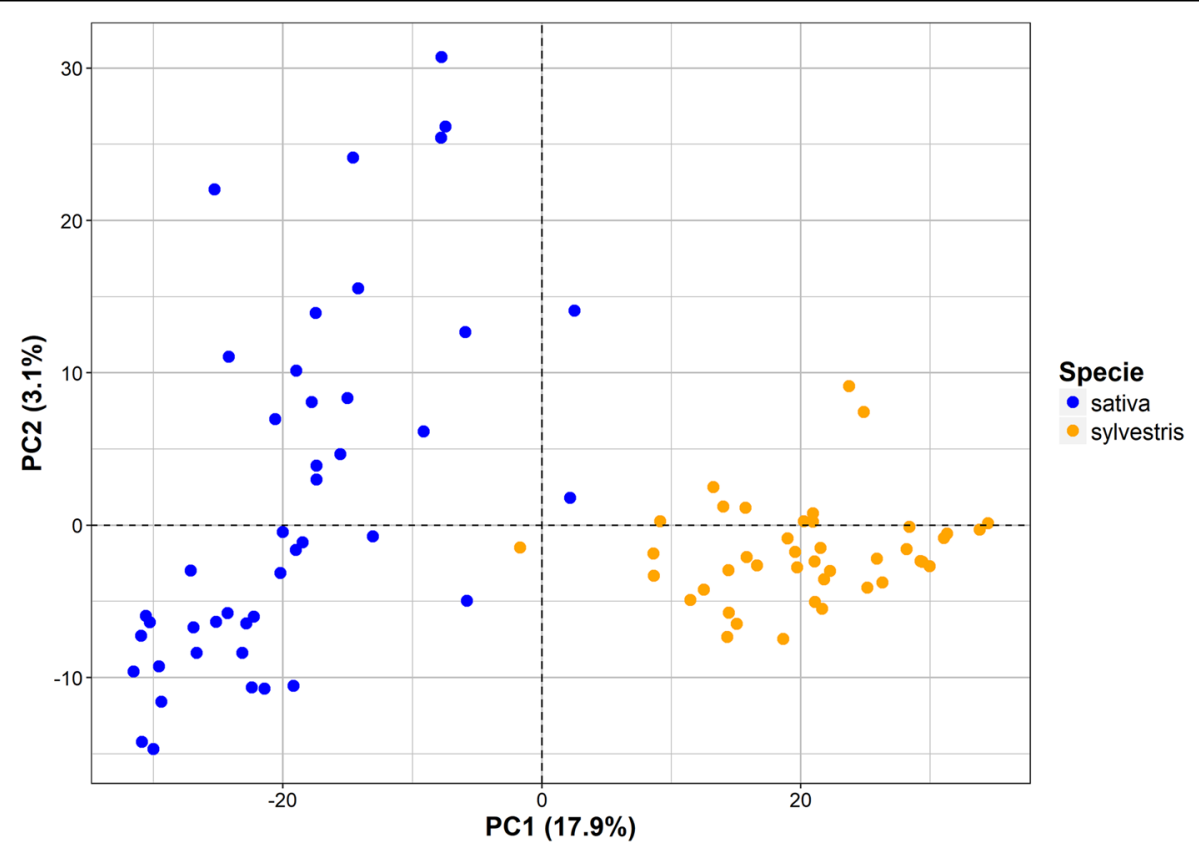

Fig. 1 Visualisation of the genetic relationships among wild and cultivated vinifera by their projection onto the first two Principal Component axes. Along each axis, the proportion of the total variance accounted by each PC is shown in parentheses

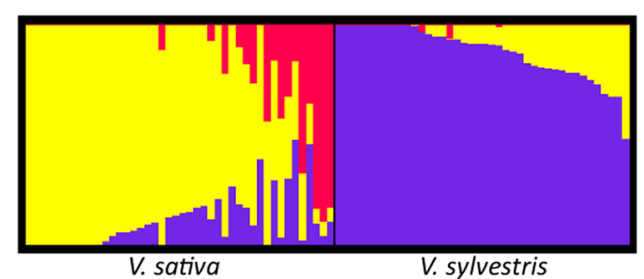

Fig. 2 Barplot of admixture proportions of wild and cultivated subpopulations, as measured by fastSTRUCTURE at $K=3$. Each individual is represented by a vertical bar, reflecting assignment probabilities to each of the three groups. K1: purple bars; K2: red bars; K3: yellow bars

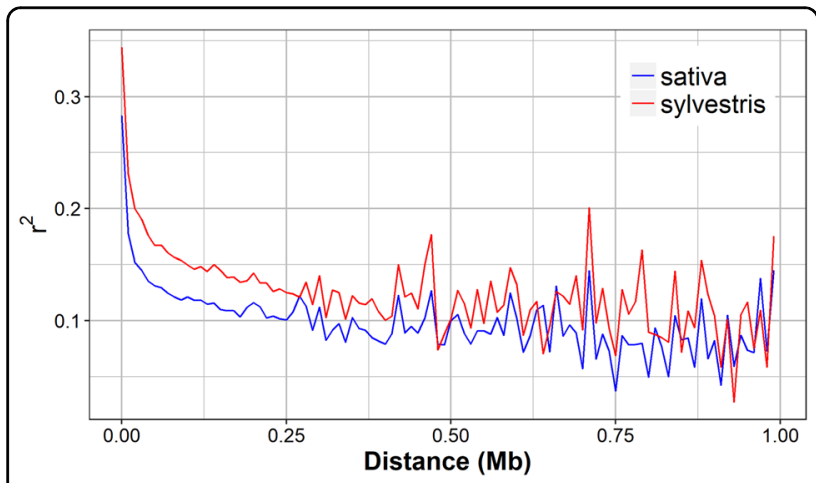

Fig. 3 Decay of LD in sativa and sylvestris separately. Each point represents the median $r^{2}$ value in sequential bins of $10 \mathrm{~Kb}$ against the physical position associated with important traits in grapevine have been identified, such as those for flower sex and berry skin colour on $\mathrm{chr} 2^{41}$, and for fruit weight on chr17 and $\operatorname{chr} 18^{42}$.

\section{Genomic differentiation between sativa and sylvestris genotypes}

Since the analysis of population structure underlined a clear separation between sativa and sylvestris accessions, we computed population differentiation statistic $\left(F_{\mathrm{ST}}\right)$ across the grapevine genome to identify genomic regions with altered allele frequency among the two $V$. vinifera subspecies. The overall level of genetic differentiation between cultivated and wild grapes was moderate $\left(F_{\mathrm{ST}}=\right.$ 0.12 ). A similar genetic divergence was reported among Western European cultivars and wild genotypes ${ }^{4}$ as well as among grapevine accessions of sativa and sylvestris from Spain ${ }^{43}$ and Morocco ${ }^{44}$. This low level of genetic differentiation suggests gene flow between cultivated and wild individuals. However, we observed a non-random distribution of divergent sites along the whole genome: the 95th percentile of the $F_{\mathrm{ST}}$ empirical distribution was > 0.27 , and no positive signals were found to pass this empirical cutoff after the permutation test (Supplementary Figure S4). Sativa and sylvestris individuals differed significantly at 2461 SNPs included in 2001 windows. More than half $(63.8 \%)$ of those variants belonged to intergenic or UTR/intron regions, whereas the remaining $26.8 \%$ and $9.4 \%$ were synonymous and nonsynonymous, 


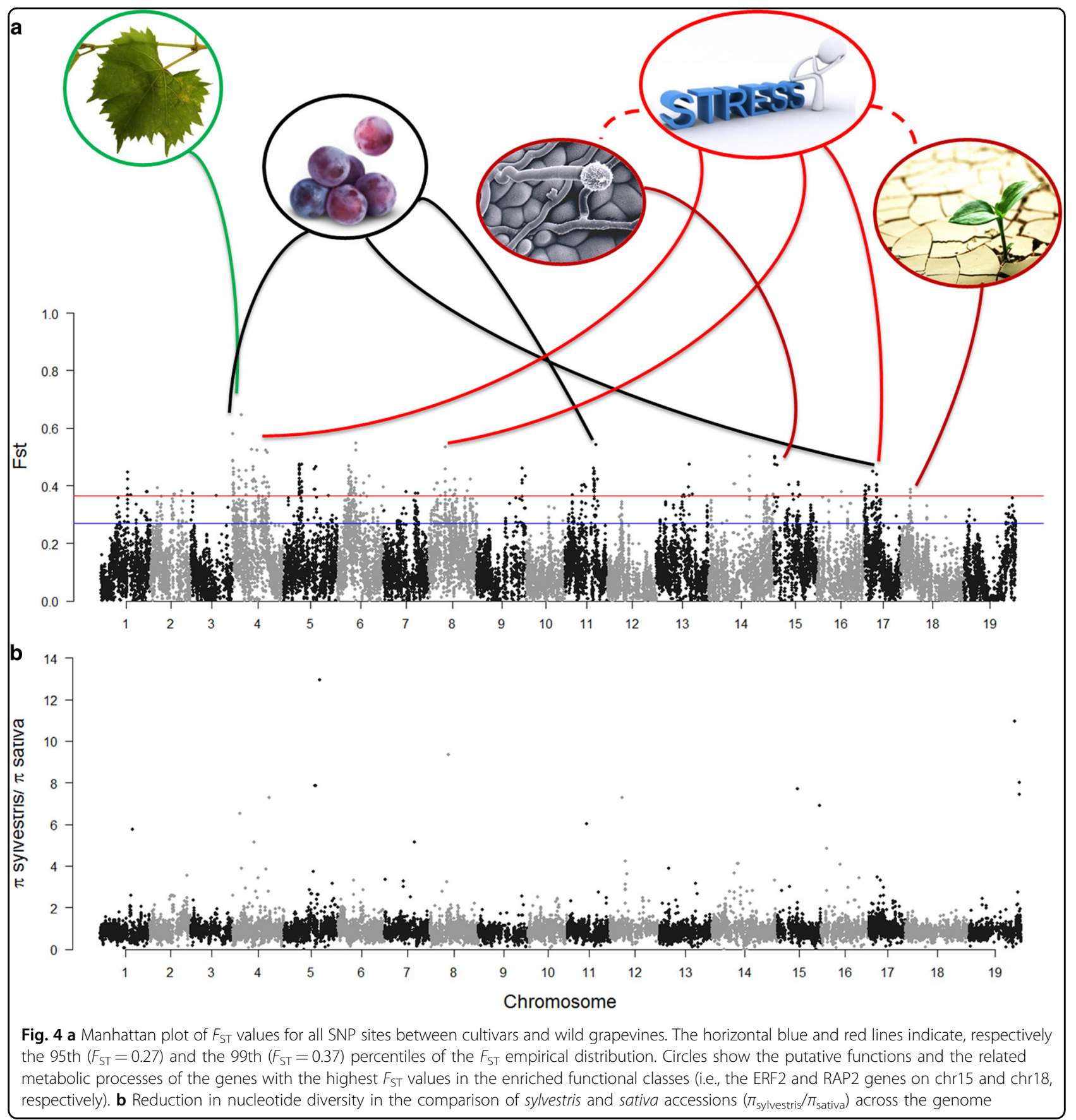

respectively. All 19 chromosomes of the grapevine genome showed divergent sites, ranging from 14 to 382 bins on chr12 and chr4, respectively (Fig. 4a).

A shift in the allele distribution within populations may result from a sweep toward fixation of a selected locus and its nearby hitchhikers ${ }^{45}$. This sweep causes a populationwide reduction in the genetic diversity around the selected locus. Therefore, nucleotide diversity $(\pi)^{46}$ was evaluated across the grapevine genome in sativa and sylvestris groups separately. As shown in Fig. 4b, the average value of the ratio $\pi_{\text {sylvestris }} / \pi_{\text {sativa }}$ was 0.89 , suggesting that $\pi$ is slightly higher in the cultivated grapevine for most of the investigated genomic regions. Several surveys in grapevine germplasm collections consisting of both cultivated and wild $V$. vinifera accessions reported this overall lower genetic diversity in the wild gene pool compared to the cultivated panel ${ }^{47}$. The wild relatives are today present in low number in isolated populations, most likely due to the 
Table 2 Functional Classes significantly differentiated between sativa and sylvestris accessions

\begin{tabular}{lllll}
\hline GO ID & Term & Annotated genes & Significant genes & $P$ value \\
\hline GO:0071704 & Organic substancemetabolic process & 1516 & 33 & 0.01596 \\
GO:0006807 & Nitrogen compoundmetabolic process & 604 & 32 & 0.01372 \\
GO:0005975 & Carbohydrate metabolic process & 148 & 9 & 0.00019 \\
GO:0055114 & Oxidation-reduction process & 143 & 8 & 0.00262 \\
GO:0009737 & Response to abscisic acid & 114 & 3 & 0.00232 \\
GO:0006952 & Defense response & 446 & 5 & 0.03388 \\
GO:0032259 & Methylation & 72 & 3 & 0.01715 \\
GO:0009607 & Response to biotic stimulus & 124 & 2 & 0.00045 \\
GO:0009651 & Response to salt stress & 50 & 2 & 0.01213 \\
GO:0010363 & Regulation of plant-type hypersensitive response & 20 & 1 & 0.0378 \\
GO:0010118 & Stomatal movement & Nucleic acid phosphodiester bond hydrolysis & 11 & 1 \\
GO:0090305 & & 9 & 0.00899 \\
\hline
\end{tabular}

anthropogenic pressure on their natural habitats and the introduction of disease-causing agents from North America at the end of the 19th century ${ }^{7}$. On the other hand, the cultivated grape has a bigger effective population size present in multiple locations, where sexual crossing and somatic mutations coupled with a massive vegetative propagation have occurred, thus accumulating and increasing genetic variability. Nevertheless, our selection of sativa accessions from a core collection may overestimate the real level of nucleotide diversity in cultivated grapevines. A drastic reduction in nucleotide diversity of sylvestris individuals $\left(\pi_{\text {sylvestris }} / \pi_{\text {sativa }}=0\right)$ was observed on chromosomes 5, 14 and 15 at genomic regions with a total of 6 SNPs monomorphic in sylvestris. At the same time, sativa showed a reduction in nucleotide diversity on chromosomes 5,12 , and 19 , where $\pi_{\text {sylvestris }} /$ $\pi_{\text {sativa }}$ had values higher than 10 . However, except for the reduction of genetic diversity in sativa on $\operatorname{chr} 19\left(F_{\mathrm{ST}}=\right.$ 0.32 ), no divergence in allele frequencies was observed for the other genomic regions with extreme values of $\pi_{\text {sylves- }}$ tris/msativa. Both cultivated and wild individuals showed low minor allele frequency at those loci $(\mathrm{MAF}<0.1)$. This reduction in nucleotide diversity in both subspecies may indicate reciprocal introgressions between wild and cultivated grapes or could reflect the occurrence of purifying selections, affecting diversity in both populations ${ }^{14}$.

Another common test used to detect signals of selection as a distortion of allele frequency and nucleotide diversity is the Tajima's $\mathrm{D}$, which compares the number of pairwise differences between individuals with the total number of segregating polymorphisms ${ }^{30}$. We observed mostly positive values of Tajima's $\mathrm{D}$ in both wild $(D: \sim 0.89)$ and cultivated $(D: \sim 1.35)$ subgroups. As reported by Riahi et al. $^{47}$, a positive value of Tajima's $D$, especially for cultivated accessions, may indicate an excess of intermediate frequency alleles in these populations. Such configuration of allele frequencies may arise by balancing selection, which maintains both alleles at the selected $\operatorname{loci}^{48}$. According to Delph \& Kelly ${ }^{49}$, this is the result of a heterozygote advantage or a spatial and temporal habitat heterogeneity. A balancing selection is in line with the high heterozygosity of grapevine genome and with the heterogeneity of uses and habitats to which $V$. vinifera is adapted.

\section{Identification of biological functions underlying the selective sweeps}

We looked at the new gene prediction v2.1 of the grapevine genome within windows of $20 \mathrm{~kb}$ around the SNPs detected as putatively under selection. Out of the 2032 predicted genes found in LD with the most significant SNPs, 1714 were annotated. Twelve functional classes were significantly enriched in the list of differentiated genes (Table 2), accounting for 109 of them (Supplementary Table S3). Most (69\%) of these genes had predicted functions related to organic compound metabolism, mainly nitrogen and carbohydrate, while $24 \%$ was assigned to functional classes involved in perception, response, and adaptation to environmental stimuli.

Out of the 109 genes in the enriched classes, 14 showed $F_{\mathrm{ST}}$ values $>0.37$ (99th percentile of the $F_{\mathrm{ST}}$ empirical distribution; Supplementary Table S3). Therefore, understanding the putative functions and the related metabolic processes of these genes is of particular relevance in the genomic comparison between sativa and sylvestris (Fig. 4). At the top of the genes list with the highest value of $F_{\mathrm{ST}}$, we identified the 'RPL5B' gene (VIT_204s0008g00050; Fig. 4 and Supplementary 
Table S3), which codifies the $60 \mathrm{~S}$ ribosomal protein L5-2. This gene could imply differences in organ development and expansion between the two subspecies. The angusta3 (ang3) mutant of $A$. thaliana for RPL5b gene displayed altered growth and development for several organs, notably leaves ${ }^{50}$. It is likely that balancing selection $\left(D_{\text {sat }}\right.$ $=1.13 ; D_{\text {syl }}=1.37$ ) has acted to promote the high morphological variation observable nowadays in leaf shape and size within and between cultivated and wild grapevines $^{51}$.

The list of genes with a significant differentiation between wild and cultivated grapevines was also particularly enriched in genes with a role in the carbohydrate metabolic processes (Table 2). For instance, the identification of the soluble starch synthase IV-1 gene (SS4; VIT_211s0065g00150; $\quad F_{\mathrm{ST}}=0.4 ; \quad$ Fig. 4) highlighted differences between the two subspecies in starch and sucrose metabolism, which is relevant for berry development $\mathrm{t}^{52}$. We also identified the nuclear transport factor 2 (NTF2) gene (VIT_217s0000g05240), which has a predicted role in response to abscisic acid (ABA), the main plant hormone promoting grape ripening ${ }^{53}$. NTF2 gene is located within the significant signature of selection on chr17, which includes candidate domestication-loci for berry size and development ${ }^{19}$. A reduction of nucleotide diversity in sativa accessions $\left(\pi_{\text {sylvestris }} / \pi_{\text {sativa }}=1.23\right)$ was observed at this locus, supporting evidence of a putative selection for berry composition and ripening traits in cultivated grapevines. Another diversified gene involved in the carbohydrate metabolism is the NADP-isocitrate dehydrogenase gene (cICDH; VIT_204s0079g00530), which catalyses the oxidative decarboxylation of isocitrate. An upregulation of the genes encoding isocitrate dehydrogenases in tobacco (Nicotiana tabacum cv Xanthi) and grape ( $V$. vinifera cv Sultanina) accompanied increased aminating activity of glutamate dehydrogenase (GDH) under stress conditions, such as salinity ${ }^{54}$.

Several of the loci highly differentiated between sativa and sylvestris were involved in response to different environmental stimuli, in agreement with recent finding $\mathrm{s}^{55}$. For instance, we identified the $10 \mathrm{kDa}$ chaperonin gene (CPN10; VIT_208s0040g01150), the 'LPA66' gene (VIT_204s0008g00480), the rhomboid-like protein 11 gene (RBL11; VIT_204s0008g03830), the desacetoxyvindoline 4-hydroxylase gene (VIT_204s0008g01360), and the FATB gene (VIT_217s0000g01100). The latter has revealed a crucial role in seed development and viability as well as in the promotion of the hypersensitive response (HR) to pathogen attack in Arabidopsis ${ }^{56}$. We also observed differences in allele frequencies at the ERF2 transcription factor (VIT_215s0021g01590) and ' $R A P 2$ ' (VIT_218s0001g05250) genes, which encode two members of the APETALA 2/ethylene-responsive element- binding factor (AP2/ERF) family. ERF proteins are ethylene-responsive element (GCC box)-binding proteins, and in tobacco, the GCC box has been found in the promoter of many defense genes ${ }^{57}$. Instead, RAP2 is a dehydration-responsive element-binding protein (DREB) with a role in plant abiotic stress responses such as highsalt stress, water deficit, and extreme temperatures ${ }^{58}$. Finally, we identified a splicing factor $3 \mathrm{~b}$ subunit 1-like gene (VIT_208s0040g00270), supporting that alternative splicing may contribute to evolutionary adaptation through the assortment of different protein isoforms as a quick response to selective pressure ${ }^{31}$.

For almost all the stress-related genes identified, we observed a modest reduction in nucleotide diversity in $s y l$ vestris $\left(\pi_{\text {sylvestris }} / \pi_{\text {sativa }} \sim 0.95\right)$, associated with a positive value of the Tajima's $\mathrm{D}\left(D_{\text {sylvestris }}=1.41\right)$. These results imply that a balancing selection was likely acting in wild populations for adaptation to several environmental changes that may have occurred in their natural habitat along river banks. Our results are in line with recent studies on the tolerance of sylvestris genotypes to different stress conditions such as pathogen attack ${ }^{11}$ or calcareous soils ${ }^{10}$, which suggest that sylvestris grapevines represent valuable resources of resilience genes or alleles likely lost during the domestication process. This would have made cultivated grapevine dependent on agricultural means such as fertilisation, irrigation, weeding, and chemical plant protection. The CPN1O and RAP2 genes represent an exception to this trend. Indeed, lower genetic diversity $\left(\pi_{\text {sylvestris }} / \pi_{\text {sativa }}\right.$ $\mathrm{CPN} 10=1.35 ; \pi_{\text {sylvestris }} / \pi_{\text {sativa }} \mathrm{RAP} 2=1.22$, respectively) was observed at these loci in sativa accessions, suggesting a putative ongoing selection for adaptive mechanisms to salt stress in the cultivated grapevine.

In addition to the GO enrichment analysis, we looked for genes identified in previous QTL mapping studies as associated with main agronomic traits in grapevine, such as berry weight, berry skin colour and flower sex (Supplementary Table S4). We found several genes of those underlying berry weight $\mathrm{QTLs}^{42}$ such as the genes for the xyloglucan endotransglycosylase (XTH; VIT_201s0150g00460) $)^{59}$, the histone deacetylase 2C (VvHD2C; VIT_206s0061g01240 $)^{60}$ and the cytochrome p450 78a3-like (CYP78A10; VIT_217s0000g05110), which has been found to regulate fruit size during tomato domestication ${ }^{61}$. Moreover, we found a signature of selection spanning from 4.7 to $5.0 \mathrm{Mb}$ on chr2 $\left(F_{\mathrm{ST}} \sim 0.31\right)$, including 4 SNPs in LD with the APT, SNP4AC and Vvib23 markers for flower sex ${ }^{62}$. We also observed differences in allele frequency $\left(F_{\mathrm{ST}}=0.36\right)$ between wild individuals, bearing colored fruits, and cultivated genotypes, composed by both colored and white varieties, at one of the MYB-type transcription factor genes on chr2 (MYB113; VIT_202s0033g00460) $^{4}$ and within other candidate genes identified at berry skin color QTLs ${ }^{63}$ (Table S4). 

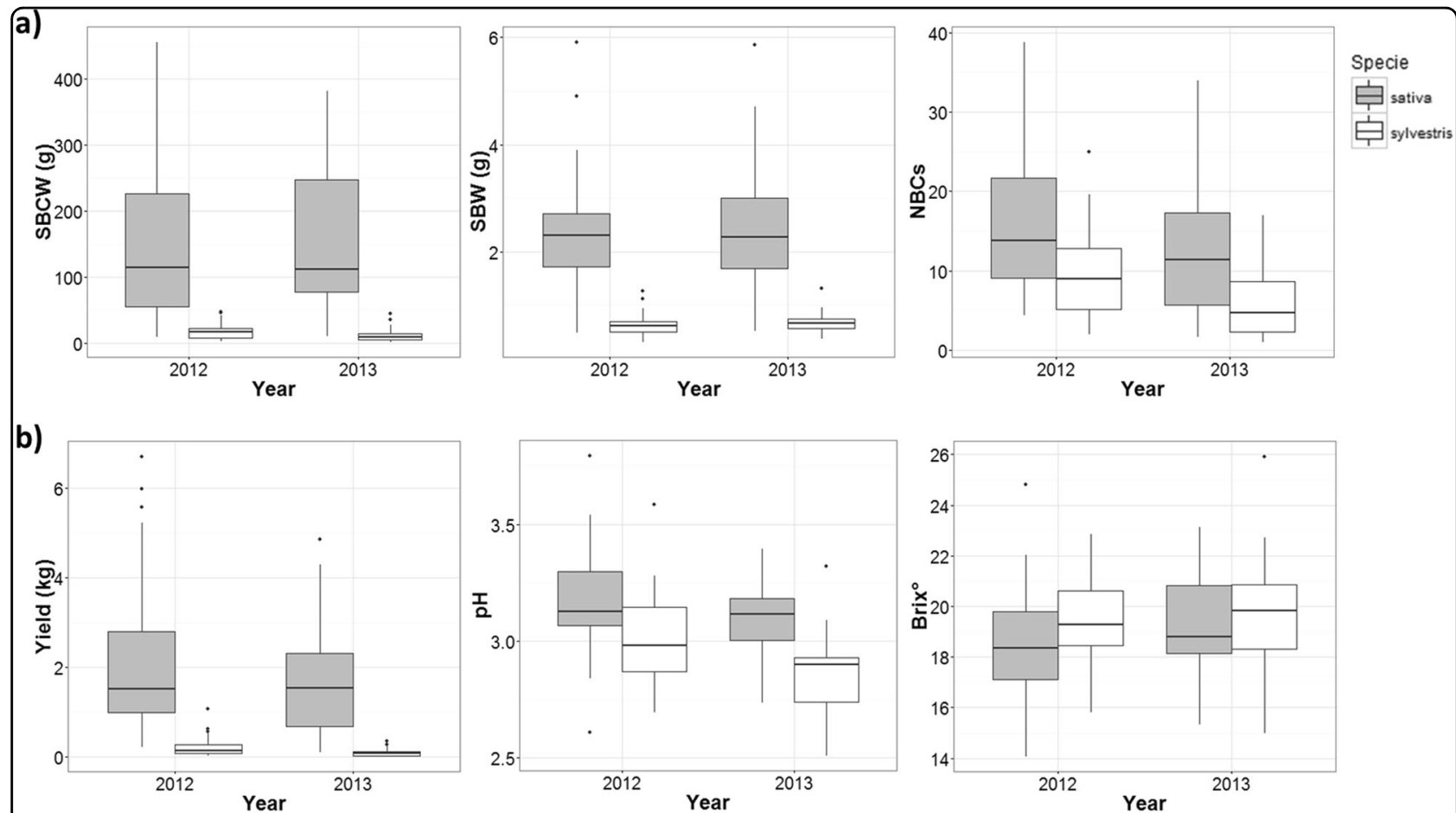

Fig. 5 Comparison of phenotypic data between cultivated (in grey) and wild (in white) individuals in the two years of measurements. (a) Box-plots of single bunch weight, single berry weight and number of bunches per plant. (b) Box-plots of yield, pH and total soluble solids.

\section{Association mapping for six domestication-related traits in grapevine}

The two vinifera subspecies exhibited an enormous phenotypic differentiation for six domestication-related traits, notably single berry weight (g; SBW), single bunch weight (g; SBCW) and berry flesh $\mathrm{pH}$ (Fig. 5a, b; Supplementary Note S1). For most traits, the cultivated individuals showed higher variability than the wild genotypes (Supplementary Figure S6 and Supplementary Table S5). In particular, sativa yielded on average numerous bunches with big and sweet berries, while sylvestris produced a few clusters with small, juiceless and acid fruits (Supplementary Note S1). These differences were more evident after estimating the Pearson's correlation coefficient $(R)$ between each pair of variables in the whole population and the two subgroups separately. While in the entire population yield was more correlated $(R \sim 0,8)$ with both SBW and SBCW than with NBCs $(R=$ 0.4 ), in sylvestris, it was highly correlated with both NBCs and SBCW rather than with SBW (Supplementary Table S6). This correlation suggests that the productivity of wild grapevine depends mainly on the number of clusters and the number of berries per bunch produced since fruit weight barely reached values higher than $1.5 \mathrm{~g}$. Furthermore, we observed a significant inverse correlation (Supplementary Table S6) for total soluble solids (Brix ${ }^{\circ}$ ) with SBW and yield in both the whole population and the cultivated grapevines. This result can be explained by the shrinkage of berries, which occurs during véraison because of the loss of water by transpiration, as well as by the decrease of sugar concentration as berry size increases ${ }^{64}$.

We attempted an association mapping study to dissect the genetic basis of the phenotype variation observed between the wild and cultivated grapevines. The strong selection occurred during domestication may have extended the LD surrounding the target loci so that the SNP density required to map domestication-related traits may be lower than that required for unselected traits ${ }^{20}$. Also, aware of the limitations in size and population structure of our association panel, we combined GWAS and population genetics, displaying here only the markertrait associations that were also identified as putative signatures of selection (Supplementary Figure S8-11). For instance, single berry weight (SBW) was significantly associated before $p$ value correction with 2 SNPs on chr6 (Supplementary Table S7), which both fell within a genomic region significantly differentiated between sativa and sylvestris individuals $\left(F_{\mathrm{ST}}=0.28\right)$. These SNPs, separated by $6.7 \mathrm{~kb}$, are in LD with five genes, among which a $\mathrm{Ca}^{2+}$ transporting ATPase endoplasmic reticulum-typelike gene (Supplementary Tables S10). This finding supports the role of calcium ion in the development of grape berries $^{65}$. 
Significant values of $F_{\text {ST }}$ were also observed for one SNP on chr15 associated with the number of bunches per plant $\left(\mathrm{NBCs} ; F_{\mathrm{ST}}=0.28\right)$, and one marker on chr14 significantly correlated with the total soluble solids $\left(\mathrm{Brix}^{\circ} ; F_{\mathrm{ST}}=0.32\right.$; Supplementary Table S7). In particular, different values of Brix $^{\circ}$ at collection were observed among the three genotypes AA (0), AB (1) and BB (2) of the marker chr14_26697249 (Supplementary Figure S13), which belongs to a long LD block of $150 \mathrm{~kb}$ (Supplementary Table S8). In this region, we identified the cytochrome p450 724b1 gene implicated in the biosynthesis of brassinosteroids (BR), whose endogenous levels increase simultaneously with berry weight and soluble solids (Brix $\left.{ }^{\circ}\right)$ at the onset of berry ripening ${ }^{66}$.

We identified the highest number of marker-trait associations for the "Species," a binary trait accounting for the level of genetic differentiation between cultivated and wild grapevine ${ }^{67}$. In particular, 34 SNPs were associated with the subspecies membership, out of which 3 SNPs on chr15 exhibited significant Bonferroni-corrected associations also with GLM-Q3 (see Supplementary Note S1; Supplementary Table S7 and Supplementary Figure S10). Notably, in LD with those three SNPs on chr15, we identified the nitrate transporter -like NRT1 gene, which has been correlated with the divergence in nitrate-use between the subspecies Oryza sativa L. indica and japonica ${ }^{68}$. According to the genome scan for signatures of selection, the GWAS test on 'species' trait led to identifying genes involved in response to environmental stresses (Supplementary Table S8). For instance, the salt overly sensitive 1 (SOS1) gene encodes a $\mathrm{Na}^{+} / \mathrm{H}^{+}$ antiporter, which is the downstream target of the Salt Overly Sensitive (SOS) signaling pathway, involved in controlling ion homoeostasis during salt stress ${ }^{69}$. We also identified the hypoxia up-regulated protein 1-like (HRE1) gene, which encodes an ERF transcription factor. HRE1 responds rapidly to oxygen deprivation by maintaining the expression of some anaerobic genes such as the alcohol dehydrogenase $(A D H)$ gene $^{70}$. Finally, the identification of the arginase gene, involved in the biosynthesis of polyamines, and the sugar transporter erd6-like 16-like gene, which encodes a monosaccharide transporter ${ }^{71}$, highlighted how sativa and sylvestris might present differences in the metabolisms of polyamines and sugars.

\section{Conclusions}

We displayed a whole-genome survey of the genetic differentiation between wild and cultivated grapevines by using population genetics approaches. An overall reduction of genetic diversity was observed within the wild panel, supporting the occurrence of an ongoing progressive decline of natural wild grapevine populations, and the necessity of developing new strategies for the characterization and conservation of $V . v$. sylvestris. Moreover, we identified several genomic regions with divergent allele frequencies between grapevine cultivars and their wild relatives. These genomic regions showed significant enrichment in functional gene classes related to responses to biotic and abiotic stresses, unraveling different putative mechanisms of adaptation to environmental changes. While grapevine cultivars are almost entirely dependent on human agricultural practices, the wild forms included in our study seem to have kept facing the permanent environmental alterations in their natural habitats. Future genome-scans using broader grapevine populations including sylvestris from their current whole worldwide distribution may confirm whether the differentiation in stress-related genomic regions is common evidence between all wild and cultivated vinifera populations, or if it is limited to the analyzed ex situ germplasm. Moreover, our study provides candidate genes for future functional genomics studies, to assess how the two forms of $V$. vinifera react under particular environmental stresses such as water deficit and pathogen attacks. In conclusion, our results support the large potential of sylvestris as a source of resilience factors in future breeding programs to deal with climate change and the increasing demand of sustainable viticulture.

\section{Acknowledgements \\ We are grateful to Giovanna Flaim for English supervision.}

\section{Authors' contributions}

A.M. conceived the study and elaborated its design, carried out the genotyping and phenotyping of the whole population, performed the analysis of LD decay, population genetics, GO enrichment and GWAS, and wrote the manuscript. D.M. participated in the study design and provided support in interpreting the results and writing the manuscript. S.L. provided lab assistance during the SNP genotyping and in the field during the trait phenotyping. D.N. provided support in interpreting the results of population genetics. M.S.G. supervised and coordinated the study, and drafted part of the manuscript. All authors read and approved the final manuscript.

\section{Author details \\ 'Department of Genomics and Biology of Fruit Crops, Research and Innovation Centre, Fondazione Edmund Mach, San Michele all 'Adige (TN), Italy. ${ }^{2}$ Computational Biology Unit, Research and Innovation Centre, Fondazione Edmund Mach, San Michele all 'Adige (TN), Italy. ${ }^{3}$ Department of Plant Sciences, University of California, Davis, CA 95616, USA. ${ }^{4}$ Center Agriculture Food Environment (C3A), University of Trento, San Michele all 'Adige (TN), Italy}

\section{Conflict of interest}

The authors declare no conflict of interest.

Supplementary Information accompanies this paper at (https://doi.org/ 10.1038/s41438-018-0041-2).

Received: 7 December 2017 Revised: 23 March 2018 Accepted: 6 April 2018 Published online: 01 July 2018

\section{References}

1. This, P., Lacombe, T. \& Thomas, M. R. Historical origins and genetic diversity of wine grapes. Trends Genet. 22, 511-519 (2006). 
2. Drori, E. et al. Collection and characterization of grapevine genetic resources (Vitis vinifera) in the Holy Land, towards the renewal of ancient winemaking practices. Sci. Rep. 7, 44463 (2017).

3. Emanuelli, F. et al. Genetic diversity and population structure assessed by SSR and SNP markers in a large germplasm collection of grape. BMC Plant Biol. 13, 39 (2013).

4. Myles, S. et al. Genetic structure and domestication history of the grape. Proc. Natl Acad. Sci. USA 108, 3530-3535 (2011).

5. De Lorenzis, G., Chipashvili, R., Failla, O. \& Maghradze, D. Study of genetic variability in Vitis vinifera L. germplasm by high-throughput Vitis18kSNP array: the case of Georgian genetic resources. BMC Plant Biol. 15, 154 (2015).

6. Marrano, A. et al. SNP-discovery by RAD-sequencing in a germplasm collection of wild and cultivated grapevines (V. vinifera L.). PLOS ONE 12, 1-19 (2017).

7. Arroyo-García, R. A. \& Revilla, E. in The Mediterranean Genetic Code-Grapevine and Olive (ed Sladonja, B.) 51-72. London: Headquarters IntechOpen Limited, (2013).

8. Di Vecchi-Staraz, M. et al. Low level of pollen-mediated gene flow from cultivated to wild grapevine: Consequences for the evolution of the endangered subspecies Vitis vinifera L. subsp. silvestris. J. Hered. 100, 66-75 (2009).

9. Doulati Baneh, H., Hassani, A. \& Abdollahi, R. Growth and physiological responses of some wild grapevine (Vitis vinifera L. ssp. sylvestris) genotypes to salinity. Bulg. J. Agric. Sci. 21, 530-535 (2015).

10. Cambrollé, J., García, J. L., Figueroa, M. E. \& Cantos, M. Physiological responses to soil lime in wild grapevine (Vitis vinifera ssp. sylvestris). Environ. Exp. Bot. 105, 25-31 (2014).

11. Duan, D. et al. Genetic diversity of stilbene metabolism in Vitis sylvestris. J. Exp. Bot. 66, 3243-3257 (2015).

12. Nielsen, R. et al. Genomic scans for selective sweeps using SNP data Genomic scans for selective sweeps using SNP data. Genome Res. 15, 1566-1575 (2005).

13. Lin, T. et al. Genomic analyses provide insights into the history of tomato breeding. Nat. Genet. 46, 1220-1226 (2014).

14. Hufford, M. B. et al. The genomic signature of crop-wild introgression in maize. PLOS Genet. 9, e1003477 (2013).

15. Huang, $X$. et al. A map of rice genome variation reveals the origin of cultivated rice. Nature 490, 497-501 (2012).

16. Bonhomme, M. et al. Genomic signature of selective sweeps illuminates adaptation of Medicago truncatula to root-associated microorganisms. Mol. Biol. Evol. 32, 2097-2110 (2015).

17. Nicolas, S. D. et al. Genetic diversity, linkage disequilibrium and power of a large grapevine (Vitis vinifera $\mathrm{L}$ ) diversity panel newly designed for association studies. BMC Plant Biol. 16, 74 (2016).

18. Chitwood, D. H. et al. A modern ampelography: a genetic basis for leaf shape and venation patterning in grape. Plant Physiol. 164, 259-272 (2014).

19. Myles, S. et al. Rapid genomic characterization of the genus Vitis. PLOS ONE 5, e8219 (2010).

20. Migicovsky, Z. et al. Patterns of genomic and phenomic diversity in wine and table grapes. Hortic. Res. 4, 17035 (2017).

21. LePaslier, M.-C. et al. The GRAPERESEQ 18K Vitis genotyping chip. In IX International Symposium on Grapevine Physiology \& Biotechnology 18. La Serena (CHL) (2013).

22. Money, D. et al. Linklmpute: Fast and Accurate Genotype Imputation for NonModel Organisms. G3 5, 2383-2390 (2015).

23. Chang, C. C. et al. Second-generation PLINK: rising to the challenge of larger and richer datasets. Gigascience 4, 7 (2015).

24. Raj, A., Stephens, M. \& Pritchard, J. K. FastSTRUCTURE: variational inference of population structure in large SNP data sets. Genetics 197, 573-589 (2014).

25. Jakobsson, M. \& Rosenberg, N. A. CLUMPP: A cluster matching and permutation program for dealing with label switching and multimodality in analysis of population structure. Bioinformatics 23, 1801-1806 (2007).

26. Rosenberg, N. A. DISTRUCT: A program for the graphical display of population structure. Mol. Ecol. Notes 4, 137-138 (2004).

27. Jombart, T. Adegenet: A R package for the multivariate analysis of genetic markers. Bioinformatics 24, 1403-1405 (2008).

28. Barrett, J. C., Fry, B., Maller, J. \& Daly, M. J. Haploview: analysis and visualization of LD and haplotype maps. Bioinformatics 21, 263-265 (2005).

29. Danecek, P. et al. The variant call format and VCFtools. Bioinformatics 27 2156-2158 (2011)

30. Tajima, F. Statistical method for testing the neutral mutation hypothesis by DNA polymorphism. Genetics 123, 585-595 (1989).
31. Vitulo, N. et al. A deep survey of alternative splicing in grape reveals changes in the splicing machinery related to tissue, stress condition and genotype. BMC Plant Biol. 14, 99 (2014).

32. Alexa, A., Rahnenführer, J. \& Lengauer, T. Improved scoring of functional groups from gene expression data by decorrelating GO graph structure. Bioinformatics 22, 1600-1607 (2006).

33. Bradbury, P. J. et al. TASSEL: Software for association mapping of complex traits in diverse samples. Bioinformatics 23, 2633-2635 (2007).

34. Turner, S. D. qqman: an R package for visualizing GWAS results using Q-Q and manhattan plots. bioRxiv 1-2 (2014).

35. Deschamps, S., Llaca, V. \& May, G. D. Genotyping-by-sequencing in plants Biology (Basel) 1, 460-483 (2012).

36. Regner, F., Stadlbauer, A., Eisenheld, C. \& Kaserer, H. Genetic relationships among Pinots and related cultivars. Am. J. Enol. Vitic. 51, 7-14 (2000).

37. Adam-Blondon, A., Martinez-Zapater, J. M. \& Kole, C. Genetics, Genomics and Breeding of Grapes St. Helier, Jersey, British Channel Islands: Science Publishers (2011).

38. Lijavetzky, D., Cabezas, J. A., Ibáñez, A., Rodríguez, V. \& Martínez-Zapater, J. M. High throughput SNP discovery and genotyping in grapevine (Vitis vinifera L.) by combining a re-sequencing approach and SNPlex technology. BMC Genom. 8, 424 (2007).

39. Velasco, R. et al. A high quality draft consensus sequence of the genome of a heterozygous grapevine variety. PLOS ONE 2, e1326 (2007).

40. Koch, E., Ristroph, M. \& Kirkpatrick, M. Long range linkage disequilibrium across the human genome. PLOS ONE 8, e80754 (2013).

41. Costantini, L., Battilana, J., Lamaj, F., Fanizza, G. \& Grando, M. S. Berry and phenology-related traits in grapevine (Vitis vinifera L.): from quantitative trait loci to underlying genes. BMC Plant Biol. 8, 38 (2008).

42. Doligez, A. et al. New stable QTLs for berry weight do not colocalize with QTLs for seed traits in cultivated grapevine (Vitis vinifera L.). BMC Plant. Biol. 13, 217 (2013).

43. De Andrés, M. T. et al. Genetic diversity of wild grapevine populations in Spain and their genetic relationships with cultivated grapevines. Mol. Ecol. 21 800-816 (2012).

44. Zinelabidine, L. H., Haddioui, A., Bravo, G., Arroyo-García, R. \& Zapater, J. M. M. Genetic origins of cultivated and wild grapevines from Morocco. Am. J. Enol. Vitic. 61, 83-90 (2010).

45. Vitti, J. J., Grossman, S. R. \& Sabeti, P. C. Detecting natural selection in genomic data. Annu. Rev. Genet. 47, 97-120 (2013).

46. Tajima, F. Evolutionary relationship of DNA sequences in finite populations. Genetics 105, 437-460 (1983).

47. Riahi, L. et al. Single nucleotide polymorphism and haplotype diversity of the gene NAC4 in grapevine. Ind. Crops Prod. 43, 718-724 (2013).

48. Charlesworth, D. Balancing selection and its effects on sequences in nearby genome regions. PLoS Genet. 2, 379-384 (2006).

49. Delph, L. F. \& Kelly, J. K. On the importance of balancing selection in plants Lynda. New Phytol. 100, 130-134 (2013).

50. Devis, D., Firth, S. M., Liang, Z. \& Byrne, M. E. Dosage sensitivity of RPL9 and concerted evolution of ribosomal protein genes in plants. Front. Plant Sci. $\mathbf{6}$ 1-12 (2015).

51. Bodor, P., Ladányi, M., Grzeskowiak, L., Grando, M. S. \& Bisztray, G. D. Ampelometric evaluation of wild grape Nitis vinifera L. ssp. sylvestris (C.C. Gmel.) Hegi) accessions in the germplasm collection of FEM-IASMA, Italy. Vitis J. Grapevine Res. 54, 213-215 (2015).

52. Agudelo-Romero, P. et al. Search for transcriptional and metabolic markers of grape pre-ripening and ripening and insights into specific aroma development in three Portuguese cultivars. PLOS ONE 8, e60422 (2013).

53. Fortes, A. M., Teixeira, R. T. \& Agudelo-Romero, P. Complex interplay of hormonal signals during grape berry ripening. Molecules 20, 9326-9343 (2015).

54. Skopelitis, D. S. et al. Abiotic stress generates ROS that signal expression of anionic glutamate dehydrogenases to form glutamate for proline synthesis in tobacco and grapevine. Plant Cell 18, 2767-2781 (2006).

55. Zhou, Y., Massonnet, M., Sanjak, J. S., Cantu, D. \& Gaut, B. S. Evolutionary genomics of grape (Vitis vinifera ssp. vinifera) domestication. Proc. Natl Acad. Sci. https://doi.org/10.1073/pnas.1709257114 (2017)

56. Raffaele, S. et al. A MYB transcription factor regulates very-long-chain fatty acid biosynthesis for activation of the hypersensitive cell death response in Arabidopsis. Plant Cell 20, 752-767 (2008).

57. Nakano, T., Nishiuchi, T., Suzuki, K., Fujimura, T. \& Shinshi, H. Studies on transcriptional regulation of endogenous genes by ERF2 transcription factor in tobacco cells. Plant Cell Physiol. 47, 554-558 (2006). 
58. Mizoi, J., Shinozaki, K. \& Yamaguchi-Shinozaki, K. AP2/ERF family transcription factors in plant abiotic stress responses. Biochim. Biophys. Acta 1819, 86-96 (2012).

59. Chervin, $\mathrm{C}$. et al. Ethylene seems required for the berry development and ripening in grape, a non-climacteric fruit. Plant Sci. 167, 1301-1305 (2004).

60. Kaps, M. L. \& Cahoon, Ga Growth and fruiting of container-grown Seyval blanc grapevines modified by changes in crop level, leaf number and position, and light exposure. Am. J. Enol. Vitic. 43, 191-199 (1992).

61. Chakrabarti, M. et al. A cytochrome P450 regulates a domestication trait in cultivated tomato. Proc. Natl Acad. Sci. USA 110, 17125-17130 (2013).

62. Fechter, I. et al. Candidate genes within a $143 \mathrm{~kb}$ region of the flower sex locus in Vitis. Mol. Genet. Genom. 287, 247-259 (2012).

63. Costantini, L. et al. New candidate genes for the fine regulation of the colour of grapes. J. Exp. Bot. 66, 4427-4440 (2015).

64. Coombe, B. G. \& McCarthy, M. G. Dynamics of grape berry growth and physiology of ripening. Aust. J. Grape Wine Res. 6, 131-135 (2000).
65. Deluc, L. G. et al. Transcriptomic and metabolite analyses of Cabernet Sauvignon grape berry development. BMC Genome 8, 429 (2007).

66. Symons, G. M. et al. Grapes on steroids. brassinosteroids are involved in grape berry ripening. Plant. Physiol. 140, 150-158 (2006).

67. Leforestier, D. et al. Genomic basis of the differences between cider and dessert apple varieties. Evol. Appl. 8, 650-661 (2015).

68. Hu, B. et al. Variation in NRT1.1B contributes to nitrate-use divergence between rice subspecies. Nat. Genet. 47, 834-838 (2015)

69. Ji, H. et al. The salt overly sensitive (SOS) pathway: Established and emerging roles. Mol. Plant 6, 275-286 (2013).

70. Licausi, F. et al. HRE1 and HRE2, two hypoxia-inducible ethylene response factors, affect anaerobic responses in Arabidopsis thaliana. Plant. J. 62, 302-315 (2010).

71. Afoufa-Bastien, D. et al. The Vitis vinifera sugar transporter gene family: phylogenetic overview and macroarray expression profiling. Bmc. Plant. Biol. 10, 245 (2010). 